\title{
Intrapartum Uterine Activity and Neonatal Outcomes: A Systematic Review
}

\author{
Adam Reynolds ${ }^{1}$, Michael Geary ${ }^{1}$, and Breda Hayes ${ }^{1}$ \\ ${ }^{1}$ Rotunda Hospital
}

May 6, 2020

\begin{abstract}
Background Increased uterine activity (UA) may not allow adequate recovery time for foetal oxygenation. Objectives To determine if increased UA during labour is associated with an increased risk of either short- or long-term neurological injury in term neonates, or with neonatal proxy measures of intrapartum hypoxia-ischemia. Search Strategy MEDLINE, CINAHL, and ClinicalTrials.gov using terms uterine activity, Excessive Uterine Activity, XSUA, Uterine hyperstimulation, and Tachysystole. Selection Criteria Any study that analysed the relationship between UA during term labour and neurological outcomes/ selected proxy neurological outcomes was eligible for inclusion. Data Collection and Analysis Outcomes from individual studies were reported in tables and presented descriptively with odds ratios (OR) and 95\% confidence intervals (CI) for dichotomous outcomes and means with standard deviations for continuous outcomes. Where group numbers were provided, ORs and CIs were calculated according to Altman. Main Results Twelve studies met the inclusion criteria. Seven studies featured umbilical artery pH as an individual outcome. Umbilical artery base excess and Apgar scores were both reported as individual outcomes in four studies. No study examined long term neurodevelopmental outcomes and only one study reported on encephalopathy as an outcome. The evidence for a relationship between UA and adverse infant outcomes was inconsistent. The reported estimated effect size varied from non-existent to clinically significant. Conclusions There is some evidence that increased UA may be a non-specific predictor of depressed neurological function in the newborn, but it is inconsistent and insufficient to support the conclusion that an association generally exists.
\end{abstract}

\section{Keywords}

Labour, Intrapartum, Fetal Monitoring, Tachysystole, Uterine Activity, Uterine Contraction

\section{Main text}

\section{Introduction}

The rationale for the proposed link between excessive uterine activity (UA) and fetal hypoxia is based on physiological studies of the haemodynamic changes that occur in the utero-placental and fetal circulations during a contraction. As a uterine contraction progresses, the amount of oxygenated blood delivered to the placenta decreases. Thus, the lowest $\mathrm{FSpO}_{2}$ values are found towards the end of a contraction and take some time to recover. Increased contraction frequencies may not allow adequate recovery time and may result in progressive reductions in fetal oxygen levels. (1-3)

There is evidence that tocolytic medications may improve fetal heart rate (FHR) abnormalities when used while emergency delivery is pending. (4) Compared to emergent delivery, tocolysis for fetal distress may 
improve umbilical artery $(\mathrm{UmA})$ base excess $(\mathrm{BE})$ values and reduce neonatal intensive care unit admission at the expense of increasing caesarean delivery. (5) A 2018 Cochrane review found several studies which showed an improvement in fetal wellbeing in response to tocolysis but concluded that, given the small sample sizes involved, "the clinical significance is unclear. (6)

Current guidelines are based on the opinion that when there is an abnormal FHR pattern and tachysystole (TS), increased UA may cause fetal hypoxia. (7) The 2014 American College of Obstetricians and Gynaecologists (ACOG) guidelines, Neonatal Encephalopathy and Neurologic Outcome, state that TS should be treated whenever it is associated with recurrent FHR decelerations or if oxytocin is being administered.

(8) The UK National Institute for Health and Care Excellence guidelines on labour management state that where the FHR pattern is suspicious or pathological any uterine hyperstimulation should be corrected. (9) There are no existing guidelines which address TS in unaugmented labour without FHR abnormalities.

The aim of this systematic review is to determine if increased UA during labour is associated with an increased likelihood of either short- or long-term neurological injury in term neonates, or with proxy measures of intrapartum hypoxia-ischemia.

\section{Methods}

The study protocol was registered with PROSPERO (CRD4201705258). The study followed the Preferred Reporting Items for Systematic Reviews and Meta-Analyses (PRISMA) statement.

\section{Search Strategy}

We searched MEDLINE, CINAHL, and ClinicalTrials.gov using terms related to UA ("uterine activity", "excessive uterine activity", "XSUA", "uterine hyperstimulation", and "tachysystole"). The publication start date was $1^{\text {st }}$ January 1996. Reference lists of relevant papers were checked to identify further papers for consideration. The results were de-duplicated using COVIDENCE (Covidence systematic review software, Veritas Health Innovation Ltd., Melbourne, Australia). The search was repeated prior to final analysis.

\section{Eligibility Criteria}

Eligibility criteria were pre-determined. Based on the anticipated heterogeneity and small size of the evidence base, we opted for permissive eligibility criteria. Any study that analysed the relationship between UA during term labour and neurological outcomes or selected proxy outcomes was eligible. The proxy outcomes were selected because they are commonly used indicators of intrapartum hypoxia-ischemia. (10) Based on incomplete reporting, conference abstracts were excluded. Studies retrieved were screened by one author and potentially eligible studies were then assessed by two team members before a final inclusion decision.

Data Extraction and Evidence Summary

A standardised form was used to extract data. Missing data was requested from authors and included if returned. Outcomes from individual studies were reported in tables and presented descriptively with odds ratios (OR) and 95\% confidence intervals (CI) for dichotomous outcomes and means with standard deviations (SD) for continuous outcomes where available. Where group numbers were provided, ORs and CIs were calculated according to Altman. (11) Results adjusted based on multivariate analysis were included as reported. A post-hoc analysis of the relationship between uterine activity and both FHR patterns and delivery mode was also performed.

Quality Assessment

The risk of bias was independently assessed by both reviewers using The Scottish Intercollegiate Guideline Network (SIGN) critical appraisal checklists but was not used to exclude any results.

Funding 
Funding was provided by The National Women and Infants Health Programme (Ireland) and the Rotunda Foundation (Registered Charity Number. 20079529).

\section{Results}

\section{Search Results and Study Selection}

Our search retrieved 1,777 citations from MEDLINE, CINAHL. (Table S1) Four additional articles were identified after review of references. The search result details are specified in supplementary material. A search of ClinicalTrials.gov did not reveal any unpublished but otherwise eligible studies. Two conference abstracts with possibly eligible results were identified but not included owing to the incomplete nature of the reports. $(12,13)$ After screening of abstracts, 21 articles were selected for full-text review. Of these, nine were excluded.(3, 14-21) (Figure 1)

\section{Study characteristics}

The characteristics of each included study are summarized in Table S2. Twelve studies met the inclusion criteria. (22-33) In five $(22,23,26,31,32)$, the primary analysis was grouped by exposure i.e. UA. In three $(24,30,33)$, cases were identified and then selectively matched to controls. For the other nine studies, all eligible patients were recruited regardless of outcome (median subject number: 1,433 [interquartile range: $720-8,008$, range: $430-50,335])$. In three $(23,25,27)$ data was collected prospectively with the primary purpose being to compare UA to fetal outcomes. In two $(34,35)(31,32)$, data was collected prospectively but the included studies were secondary analyses. The data collected pertained to labours from 1993 to 2015. Subjects were recruited in North America (7 studies), Sweden, Turkey, Italy, The Netherlands, and Ireland (1 study each).

Included labours were exclusively spontaneous and unaugmented (26), exclusively induced/augmented (23, $25,27,32)$, or mixed $(22,24,28-31,33)$.

One study (29) exclusively used measurements from intra-uterine pressure catheters (IUPC). Three studies $(27,32,33)$ used external tocography data only. Two studies $(23,30)$ reported using IUPC and external tocography but mainly external measurements. Six studies $(22,24-26,28,31)$ did not specify the measurement technology.

Three studies (28-30) used automated analysis. Four studies $(22,26,27,33)$ used documentation recorded during labour by the attending midwives The remainder $(23-25,31,32)$ reported retrospectively analysed recordings, the interpretation of which was stated to be blinded in all except one (32).

The period of labour analysed varied significantly between studies. Three $(26,27,33)$ did not explicitly state the period included. Two $(25,32)$ analysed the entire period from induction to delivery. One (24) analysed data from the first 4 hours after induction. The remainder analysed a period leading up to delivery (Bakker: last hour of $1^{\text {st }}$ stage and total $2^{\text {nd }}$ stage, Hamilton: 4h, Smith: [?]4h, Heuser: [?]2h, Jonsson: 2h, Palanisamy: 1h).

Eleven studies featured contraction rate as an individual exposure, with one (27) reporting rate only as part of a combined exposure with contraction duration. All studies apart from Bakker et al. reported contraction rate as either a dichotomised or categorised variable. Two studies $(29,30)$ included contraction rate as a continuous variable. All studies reported dichotomised outcomes only apart from one study (27) which reported $\mathrm{UmA} \mathrm{pH}$ and $\mathrm{BE}$ as continuous measures.

Six of the ten included studies published after 2008 adhered precisely or closely to the ACOG 2008 definition of TS i.e. more than five contractions in 10 minutes, averaged over a 30-minute window. Of the four that did not follow the ACOG definition: Hayes et al. referred to 15-minute contraction rates $>7$, Mutli Meydanli et al. reported a composite exposure which included the ACOG definition along with any contraction longer 
than 2-minutes, Bofill et al. required two consecutive 10-minute windows with [?]6 contractions each, and Stewart et al. reported the maximum 10-minute contraction rate.

Two studies $(23,29)$ included non-composite exposures besides contraction rate. Stewart et al. reported the number of contractions $>120 \mathrm{~s}$. Bakker et al. reported on relaxation time as well as contraction duration, amplitude, and surface (mean and total), as well as Montevideo units, and active planimeter units (mean).

\section{Risk of bias within studies}

Of the twelve included studies, three were classed as at high risk of bias, six were classed as at medium risk, and three were classed as at low risk. (Tables S3-S14).

\section{Synthesis of results}

The results of the individual studies are summarized in Table S15.

Neurodevelopmental Outcome

No studies were found which reported neurodevelopmental outcomes in relation to UA.

Hypoxic Ischemic Encephalopathy (HIE)

One study reported HIE as an outcome. In Hayes et al. contraction rates greater than 7 in 15 minutes were associated with an increased risk of neonatal encephalopathy (TS: 87/187 [46.5\%], No TS: 120/457 [26.3\%], OR: 2.44 [95\% CI: 1.71-3.48], aOR: 2.07 [95\% CI: 1.13 to 3.81]). (33)

Umbilical Artery $\mathrm{pH}$

Seven studies featured UmA pH as an individual outcome. Three rejected and four retained the null hypothesis that UA is not associated with UmA pH levels. Jonsson et al. compared cases with UmA pH $<7.05$ to controls with pH[?]7.05 and 5-minute Apgar scores [?]5. (24) Tachysystole (TS) in the last two hours before delivery was found to be associated with the likelihood of an adverse outcome (TS: 84/127 [66.1\%], No TS: 132/469 [28.1\%], OR: 4.99 [95\% CI: 3.28-7.58]). Mutlu Meydanli et al. found that TS was associated with a 7.1 (95\% CI: 1.3-38.7) times increase in the relative risk of a UA pH [?]7.15 in women after misoprostol induction (Bishop's score [?]4). (25) Bakker et al. found that UmA pH levels [?]7.11 were associated with higher contractions per 10-minutes as measured by intrauterine pressure catheters both in the first (mean: 5.0 [SD: 0.7] versus 4.8 [0.7], $\mathrm{p}=0.006$ ) and second stages (mean: 5.5 [SD: 0.9] versus 5.2 [0.9], $\mathrm{p}=0.002$ ). (29) Bofill et al. reported that TS after cervical ripening was not associated with mean UmA pH levels ([?]3 episodes: 7.22 [SD: 0.08], No episodes: 7.22 [SD: 0.08], $\mathrm{p}=0.435$ ), or with the likelihood of a UmA pH $<7.0$ (TS: 3/131 [2.3\%], No TS: 8/631 [1.3\%], OR: 1.83 [95\% CI: 0.48-6.97]). Stewart et al. reported that the maximum contraction rate in the first four hours after induction was not associated with an increased risk of acidosis ([?]4: 1/152 [1\%], 5: 4/179 [2\%], 6: 2/134 [1\%], [?]7: 6/119 [5\%], p for trend=0.06, OR for >5: 2.20 [95\% CI: 0.71-6.81]). Mussi et al. found that the presence of TS or prolonged contractions was not linearly correlated with $\mathrm{UmA} \mathrm{pH}(\mathrm{r}=0.006, \mathrm{p}=0.8)$. (27) Palanisamy et al. found that TS in the last hour of labour was not associated with UmA pH levels (TS: 9/513 [1.8\%], No TS: 140/8067 [1.7\%], OR: 1.01 [95\% CI: $0.51-2.00])$. (31)

\section{Umbilical Artery Base Excess}

Four studies tested whether UmA BE was linked to UA with two rejecting the null hypothesis. Mussi et al. found that the presence of TS or prolonged contractions was not linearly correlated with the UmA BE $(\mathrm{r}=0.15, \mathrm{p}=0.07)$. Hamilton et al. compared labours with UA BE $<-12$ to labours with UA BE $>-8$. The found that TS was associated with acidaemia (TS: 138/1215 [11.4\%], No TS: 178/2105 [8.5\%], OR: 1.39 [95\% CI: 1.10-1.75]). (30) In those with TS, the duration of TS was not associated with metabolic acidosis. Bofill et al. found that labours with [?]3 20-minute episodes of TS did not have lower mean BE values than labours without TS (mean BE: -6.81 [SD: 4.18] versus -6.57 [4.0], $\mathrm{p}=0.535$ ). Palanisamy et al. reported that 
TS in the last hour before delivery was more common in labours with a BE [?]-8 (TS: 33/513 [6.4\%], No TS: 315/8067 [3.9\%], OR: 1.69 [95\% CI: 1.17-2.45]).

Umbilical Artery Lactate

One study reported UmA lactate values. Palanisamy et al. observed that UmA lactate values [?]4 were more common when the last hour before delivery was complicated by TS (TS: 173/513 [33.7\%], No TS: 2032/8067 [25.2\%], OR: 1.51 [95\% CI: $1.25-1.83])$.

\section{Apgar Score}

Four of nine cohort studies included Apgar score as an individual outcome. One rejected and three retained the null hypothesis. Heuser at al. reported that documentation of $>5$ contractions in 10 minutes averaged over 30 minutes was associated with an increased rate of 5-minute Apgar scores < 7 (TS: 61/5363 [1.1\%], No TS: $336 / 44972$ [0.7\%], OR: 1.53 [95\% CI: 1.16-2.01]). (22) Stewart et al. reported that maximum contraction rate was not associated with an increased risk of a five-minute Apgar [?] ([?]4: 0/152 (0\%), 5: 0/134 (0\%), 6: $1 / 179(1 \%)$, [?]7: 0/119 (0\%), p for trend=0.86). (23) Ahmed at al. found that TS (> 5 contractions in 10 minutes averaged over a 30-minute window) was not associated with an Apgar score $<7$ at 5 minutes (No TS: $14 / 7118$ [0.2\%], TS: 2/890 [0.2\%], $\mathrm{p}=0.897$ ). (26) Bofill et al. reported that TS was not associated with median 5-minute Apgar scores ([?]3 TS episodes: 9 [IQR: 8-9], No TS: 9 [IQR: 8-9], p=0.502) or with the risk of 5-minute Apgar scores [?]3 ([?]3 TS episodes: 0/131 [0.0\%], No TS: 3/631 [0.5\%]). (32)

\section{Composite Outcomes}

Two studies featured composite outcomes which were composed of eligible outcomes but could not be separated based on available data. Smith et al. compared cases with an UmA BE [?]10mmol/L or a 5-minute Apgar score [?]6 to controls without those characteristics. Using automated analysis of external tocography recordings from up to 4 hours before delivery, they found that TS regardless of duration was not more common in cases than controls (No TS: 66/5095 [1.3\%], Any TS: 11/1139 [1.0\%], p=0.45). (28) Mussi et al. found that TS or prolonged contractions were not associated with either a UmA pH $<7.1$ or a UmA BE >-10 (No TS: $11 / 339$ [3.2\%], TS: 5/91 [5.5\%], p=0.35)

Figure 2 shows unadjusted odds ratios for each dichotomous outcome in the presence of TS. Mutlu Meydanli et al. was excluded from the graph as group numbers were not reported.

\section{Fetal Heart Rate Assessments}

Three of the twelve included studies compared FHR patterns in labours with TS to those without TS. All rejected the null hypothesis. Stewart et al. reported that the maximum 10-minute contraction rate was related to the presence of variable, late, or prolonged decelerations (Any decelerations: [?]4: 61/152 [40\%], 5: 88/179 [49\%], 6: 77/134 [57\%], [?]7: 69/119 [58\%] p for trend <0.0.001, OR for >5: 1.67 [95\% CI: 1.20-2.32]). Ahmed et al. found that the rate of non-reassuring FHR (NRFHR) traces in labours with TS was higher than those labours without TS (TS: 38/890 [4.3\%], no TS: 179/7118 [2.5\%], OR: 1.73 [95\% CI: 1.21-2.47]). Bofill et al. found higher rates of NRFHR patterns with TS compared to without ([?]3 TS episodes: 28/131 [21\%], No episodes: 91/631 [14\%], OR: 1.61 [95\% CI: 1.01-2.59]).

Uterine Activity and Delivery Methods

Three of the included studies reported on TS in relation to caesarean delivery for any indication. None found a statistically significant association. Heuser at al. reported that TS for any half hour period in the last two hours of labour was not associated with caesarean delivery (TS: 670/5363 [12.5\%], No TS: 5240/44972 [11.7\%], OR: 1.08 [95\% CI: 0.99-1.18]). Stewart at al. reported that TS in the first four hours after induction was not associated with caesarean delivery (TS: 43/253 [17.0\%], No TS: 66/331 [19.9\%], OR: 0.82 [95\% CI: 0.54-1.26]). Bofill et al. found that TS at any stage after induction was not associated with caesarean delivery (TS: $37 / 131$ [28.2\%], No TS: 215/631 [34.1\%], OR: 0.76 [95\% CI: 0.50-1.15]). 
Two studies reported data on caesarean delivery for FHR trace findings. Ahmed et al. found TS in spontaneous unaugmented labours to be associated with an increased risk of caesarean delivery for NRFHR traces (TS: 58/890 [6.5\%], No TS: 318/7118 [4.5\%], OR: 1.49 [95\% CI: 1.12-1.99]). Mutlu Meydanli et al. reported a statistically insignificant increase in the risk of caesarean delivery for NRFHR traces (RR: 2.4 [95\% CI: $0.6-9.4])$.

Heuser et al. found that TS was associated with an increased likelihood of operative vaginal delivery (TS: 682/5363 [12.7\%], No TS: 3491/44972 [7.8\%], OR: 1.73 [95\% CI: 1.59-1.89]).

\section{Results for Exposures Other Than Contraction Rate}

With regard to the last hour of the first stage, Bakker at al. found that $\mathrm{UmA} \mathrm{pH}<7.11$ was associated with average relaxation times (cases: $51 \mathrm{~s} \pm 23$, controls: $63 \mathrm{~s} \pm 35, \mathrm{p}<0.001$ ), average Montevideo units (cases: $261 \pm 86$, controls: $236 \pm 97, \mathrm{p}=0.02$ ), and average active planimeter units (cases: $9014 \pm 2461$, controls: $8379 \pm 2740, \mathrm{p}=0.04$ ), but not with average contraction duration (cases: $87 \mathrm{~s} \pm 9$, controls: $87 \mathrm{~s} \pm 10, \mathrm{p}=0.68$ ), average contraction amplitude (cases: $54 \mathrm{mmHg} \pm 16$, controls: $51 \mathrm{mmHg} \pm 19, \mathrm{p}=0.165$ ), or average contraction surface (cases: $1875 \mathrm{mmHg}^{*} \mathrm{~s} \pm 555$, controls: $1798 \pm 600, \mathrm{p}=0.26$ ). Similar results were reported for the second stage.

\section{Discussion}

\section{Main Findings}

The evidence for a relationship between UA and adverse infant outcomes is inconsistent. The reported effect sizes vary from small and not statistically significant to highly clinically significant.

\section{Strengths and Limitations}

Individual Studies

Only one study reported direct measures of neurological function in neonates and no study reported longterm neurological outcomes. These interactions are inherently difficult to assess because adverse outcomes of these types are rare and often require long-term follow-up.

As pre-specified in our protocol, NICU admission was excluded as an outcome. Admission to the NICU was reported either as an individual or as part of a composite outcome in three of the included studies. (22, 26,31 ) The group of babies admitted to NICU is heterogenous and likely significantly composed of babies conditions not associated with neurological function.

One study explicitly excluded neonates with encephalopathy, potentially introducing significant selection bias. Selection criteria for studies of intrapartum monitoring should allow for the inclusion of labours with negative outcomes including HIE and fetal death.

None of the included articles reported sample size calculations. Since the relevant adverse outcomes are rare and assuming modest estimated effect sizes, several of the included studies were underpowered for the variables of interest to this review.

When trying to establish a causative relationship, controlling for confounding variables through multivariate analysis should be based on a model of the mechanisms of the purported relationships between the exposure, the outcome, and the potential confounder. (34) Oxytocin and nulliparity are both associated with increased UA. $(14,35)$ Therefore, in a study of UA and neonatal outcomes, it is not usually appropriate to control for these factors since, according to the hypothesis, they may be causally related to the outcome via their effect on uterine activity. Some included studies e.g. Heuser et al. reported multivariate analyses which included these variables.

Chorioamnionitis, oligohydramnios, pre-eclampsia and the duration of labour have all been associated with both increased UA and adverse neonatal outcomes. $(22,26,36,37)$ For the most part, the connection between 
these factors and neonatal outcomes is not plausibly via their effect on tachysystole. Therefore, it may be appropriate to control for these factors when analysing the influence of UA on neonatal outcomes. They were not considered in the included studies.

Review Level

The permissive eligibility criteria for this review allowed a comprehensive survey of the literature. This approach precluded metanalysis. However, given the disparate nature of the evidence base, valid metanalysis would not have been possible even with a more focused approach.

The assessment of bias in the individual studies was limited. Current tools for assessing bias in noninterventional studies are not robust. (38)

Publication bias is common in systematic reviews and may particularly affect observational studies. (39) Since publication bias is difficult to assess accurately and owing to the heterogeneity of the included studies, no formal assessment was attempted. Study registration and replication have been proposed as solutions to publication bias and selective reporting in observational studies. $(40,41)$ None of the included trials had protocols registered at ClinicalTrials.gov. No replication studies were found.

\section{Interpretation}

Most commonly UA was defined in terms of rate only, as a dichotomous variable and without regard to labour progress. This model is partly based on unavoidable limitations of external tocography but is nonetheless a reductive view of UA which may obscure the effect of increased UA on neonatal outcomes.

The aim of this review was not to assess the impact of UA levels on FHR patterns. However, it is important to consider how interventions for FHR abnormalities might affect the relationship between UA and neonatal outcomes. Three included studies reported increased rates of FHR pattern abnormalities in labours with TS and another (22) reported worsening of FHR traces to be temporally related to TS. In Bofill et al. labours with TS were more likely to result in caesarean delivery for FHR abnormalities. If increased UA leads to fetal distress, interventions for fetal distress such as caesarean delivery might lessen the impact of increased UA and the observed effect on outcome could be weakened. Therefore, differences in the management of TS and/or fetal distress are among the possible explanations for the disparity in reported results. Future studies should report on delivery methods and their indications, as well as any intrauterine resuscitation administered so that these measures can be taken into account.

As an individual marker, it is unlikely that UA can predict the condition of a fetus after delivery. It is possible that increased UA on its own is not typically sufficient to produce significant fetal hypoxia-ischemia, but that in concert with other factors such as placental insufficiency or prolonged labour it may contribute to adverse neonatal outcomes.

\section{Conclusions}

Based on current evidence, tachysystole is common and mostly does not result in neonatal complications. There is inconsistent evidence to support the hypothesis that increased UA is associated with neonatal markers of intrapartum hypoxia-ischemia and depressed neurological function in the newborn.

\section{Disclosure of Interests}

The authors have no competing interests to disclose. 


\section{Funding}

Funding for this project was provided by The National Women and Infants Health Programme (Ireland) and the Rotunda Foundation (Registered Charity Number. 20079529). The Rotunda Foundation is a registered charity (CHY20091) and the official fundraising arm of the Rotunda Hospital. Neither funding organisation played a role in the conception or execution of the study.

\section{Contribution to Authorship}

AJR and $\mathrm{BCH}$ contributed to study design, data collection, interpretation of data, and drafting the report; MPG contributed to study design, interpretation of data, and drafting the report. AJR is guarantor.

\section{References}

1. Peebles DM, Spencer JA, Edwards AD, Wyatt JS, Reynolds EO, Cope M, et al. Relation between frequency of uterine contractions and human fetal cerebral oxygen saturation studied during labour by near infrared spectroscopy. Br J Obstet Gynaecol. 1994;101(1):44-8.

2. Biggio J. 813: Effect of uterine contraction frequency on fetal oxygen saturation and neonatal outcome. American Journal of Obstetrics \& Gynecology.212(1):S391-S2.

3. Simpson KR, James DC. Effects of oxytocin-induced uterine hyperstimulation during labor on fetal oxygen status and fetal heart rate patterns. Am J Obstet Gynecol. 2008;199(1):34 e1-5.

4. Kulier R, Gulmezoglu AM, Hofmeyr GJ, Van Gelderen CJ. Betamimetics in fetal distress: randomised controlled trial. J Perinat Med. 1997;25(1):97-100.

5. Briozzo L, Martinez A, Nozar M, Fiol V, Pons J, Alonso J. Tocolysis and delayed delivery versus emergency delivery in cases of non-reassuring fetal status during labor. J Obstet Gynaecol Res. 2007;33(3):266-73.

6. Leathersich SJ, Vogel JP, Tran TS, Hofmeyr GJ. Acute tocolysis for uterine tachysystole or suspected fetal distress. Cochrane Database Syst Rev. 2018;7:CD009770.

7. Hobson SR, Abdelmalek MZ, Farine D. Update on uterine tachysystole. J Perinat Med. 2019;47(2):152-60.

8. Executive summary: Neonatal encephalopathy and neurologic outcome, second edition. Report of the American College of Obstetricians and Gynecologists' Task Force on Neonatal Encephalopathy. Obstet Gynecol. 2014;123(4):896-901.

9. Intrapartum Care: Care of Healthy Women and Their Babies During Childbirth. Intrapartum Care: Care of Healthy Women and Their Babies During Childbirth. National Institute for Health and Care Excellence: Clinical Guidelines. London2014.

10. Azzopardi D, Brocklehurst P, Edwards D, Halliday H, Levene M, Thoresen M, et al. The TOBY Study. Whole body hypothermia for the treatment of perinatal asphyxial encephalopathy: a randomised controlled trial. BMC Pediatr. 2008;8:17.

11. Altman DG. Practical Statistics for Medical Research: Chapman \& Hall/CRC; 1990.

12. Zahedi-Spung L, Stout M, Woolfolk C, Litz T, Macones GA, Cahill AG. 764: Second stage tachysystole is associated with adverse neonatal outcomes. American Journal of Obstetrics \& Gynecology. 2019;220(1):S500S1. 
13. Bergel R, Borovich A, Gurel R, Cohen N, Chen R, Wiznitzer A, et al. 416: Does tachysystole influence maternal and neonatal outcomes during induction of labor? American Journal of Obstetrics \& Gynecology. 2018;218(1):S254.

14. Crane JM, Young DC, Butt KD, Bennett KA, Hutchens D. Excessive uterine activity accompanying induced labor. Obstet Gynecol. 2001;97(6):926-31.

15. Kunz MK, Loftus RJ, Nichols AA. Incidence of Uterine Tachysystole in Women Induced with Oxytocin. Journal of Obstetric, Gynecologic \& Neonatal Nursing. 2013;42(1):12-8.

16. Zimmer EZ, Paz Y, Copel JA, Weiner Z. The effect of uterine contractions on intrapartum fetal heart rate analyzed by a computerized system. Am J Obstet Gynecol. 1998;178(3):436-40.

17. Jonsson M, Norden-Lindeberg S, Ostlund I, Hanson U. Metabolic acidosis at birth and suboptimal care-illustration of the gap between knowledge and clinical practice. BJOG. 2009;116(11):1453-60.

18. Pacheco LD, Rosen MP, Gei AF, Saade GR, Hankins GD. Management of uterine hyperstimulation with concomitant use of oxytocin and terbutaline. Am J Perinatol. 2006;23(6):377-80.

19. Selo-Ojeme D, Rogers C, Mohanty A, Zaidi N, Villar R, Shangaris P. Is induced labour in the nullipara associated with more maternal and perinatal morbidity? Arch Gynecol Obstet. 2011;284(2):337-41.

20. Simpson KR. Excessive uterine activity during labor. MCN Am J Matern Child Nurs. 2008;33(6):396.

21. Frey HA, Tuuli MG, Roehl KA, Odibo AO, Macones GA, Cahill AG. Can contraction patterns predict neonatal outcomes? J Matern Fetal Neonatal Med. 2014;27(14):1422-7.

22. Heuser CC, Knight S, Esplin MS, Eller AG, Holmgren CM, Manuck TA, et al. Tachysystole in term labor: incidence, risk factors, outcomes, and effect on fetal heart tracings. Am J Obstet Gynecol. 2013;209(1):32 e1-6.

23. Stewart RD, Bleich AT, Lo JY, Alexander JM, McIntire DD, Leveno KJ. Defining uterine tachysystole: how much is too much? Am J Obstet Gynecol. 2012;207(4):290 e1-6.

24. Jonsson M, Norden-Lindeberg S, Ostlund I, Hanson U. Acidemia at birth, related to obstetric characteristics and to oxytocin use, during the last two hours of labor. Acta Obstet Gynecol Scand. 2008;87(7):745-50.

25. Mutlu Meydanli M, Caliskan E, Haberal A. Prediction of adverse outcome associated with vaginal misoprostol for labor induction. Eur J Obstet Gynecol Reprod Biol. 2003;110(2):143-8.

26. Ahmed AI, Zhu L, Aldhaheri S, Sakr S, Minkoff H, Haberman S. Uterine tachysystole in spontaneous labor at term. J Matern Fetal Neonatal Med. 2016;29(20):3335-9.

27. Mussi S, Incerti M, Plevani C, Ghidini A, Pezzullo JC, Locatelli A. Effect of oxytocin during labor on neonatal acidemia. J Matern Fetal Neonatal Med. 2016;29(19):3098-103.

28. Smith S, Zacharias J, Lucas V, Warrick PA, Hamilton EF. Clinical associations with uterine tachysystole. J Matern Fetal Neonatal Med. 2014;27(7):709-13.

29. Bakker PC, Kurver PH, Kuik DJ, Van Geijn HP. Elevated uterine activity increases the risk of fetal acidosis at birth. Am J Obstet Gynecol. 2007;196(4):313 e1-6.

30. Hamilton E, Warrick P, Knox E, O'Keeffe D, Garite T. High uterine contraction rates in births with normal and abnormal umbilical artery gases. J Matern Fetal Neonatal Med. 2012;25(11):2302-7.

31. Palanisamy A, Lopez J, Frolova A, Macones G, Cahill AG. Association between Uterine Tachysystole during the Last Hour of Labor and Cord Blood Lactate in Parturients at Term Gestation. Am J Perinatol. 2019;36(11):1171-8. 
32. Bofill JA, Darby MM, Castillo J, Sawardecker SU, Magann EF, Morrison JC. Tachysystole Following Cervical Ripening and Induction of Labor Is Not Associated with Adverse Outcomes. Gynecol Obstet Invest. 2017;82(5):487-93.

33. Hayes BC, McGarvey C, Mulvany S, Kennedy J, Geary MP, Matthews TG, et al. A case-control study of hypoxic-ischemic encephalopathy in newborn infants at $>36$ weeks gestation. Am J Obstet Gynecol. 2013;209(1):29.e1-.e19.

34. McNamee R. Confounding and confounders. Occupational and Environmental Medicine. 2003;60(3):22734.

35. Arulkumaran S, Gibb DM, Lun KC, Heng SH, Ratnam SS. The effect of parity on uterine activity in labour. Br J Obstet Gynaecol. 1984;91(9):843-8.

36. Strasser SM, Kwee A, Visser GH. Spontaneous tachysystole as sign of serious perinatal conditions. J Matern Fetal Neonatal Med. 2010;23(7):736-41.

37. Sandstrom A, Altman M, Cnattingius S, Johansson S, Ahlberg M, Stephansson O. Durations of second stage of labor and pushing, and adverse neonatal outcomes: a population-based cohort study. J Perinatol. 2017;37(3):236-42.

38. Bero L, Chartres N, Diong J, Fabbri A, Ghersi D, Lam J, et al. The risk of bias in observational studies of exposures (ROBINS-E) tool: concerns arising from application to observational studies of exposures. Syst Rev. 2018;7(1):242.

39. Onishi A, Furukawa TA. Publication bias is underreported in systematic reviews published in highimpact-factor journals: metaepidemiologic study. J Clin Epidemiol. 2014;67(12):1320-6.

40. de Jonge P, Conradi HJ, Thombs BD, Rosmalen JG, Burger H, Ormel J. Prevention of false positive findings in observational studies: registration will not work but replication might. J Epidemiol Community Health. 2011;65(2):95-6.

41. Loder E, Groves T, MacAuley D. Registration of observational studies. BMJ. 2010;340:c950.

\section{Hosted file}

Intrapartum Uterine Activity and Neonatal Outcomes (Figures and Tables).docx available at https://authorea.com/users/318969/articles/448785-intrapartum-uterine-activity-andneonatal-outcomes-a-systematic-review 